\title{
Clases de Educación Física activas mediante evaluación SOFIT
}

Javier A. Hall-López'1, Paulina Y. Ochoa-Martínez'1, Juan Carlos González-Terrazas ${ }^{1}$ y Eduardo J. Fernández-Ozcorta ${ }^{2}$

'Facultad de Deportes, Universidad Autónoma de Baja California (UABC), México

2 Grupo de Investigación EMOTION, Universidad de Huelva. Email: eduardo.fernandez@dempc.uhu.es

RESUMEN: El presente estudio tuvo como finalidad implementar un programa de formación a 34 estudiantes de la Licenciatura en Actividad Física y Deporte de la Escuela de Deportes de la Universidad Autónoma de Baja California (México), con el objeto de mejorar las el tiempo de actividad e intensidad de las clases de EF impartidas por estos estudiantes. Para la idoneidad del programa de formación se evaluaron las variables intensidad, índice de actividad física moderada a vigorosa y contexto evaluada mediante el System for Observing Fitness Instruction Time (SOFIT; McKenzie, Sallis, y Nader, 1991). Tras el programa de capacitación los resultados, tras impartir 34 sesiones de EF a niños de edad escolar, se encontró que la intensidad moderada a vigorosa se encontraba por encima del 50\%. Asimismo, se entró que el tiempo aumento en tareas relacionadas con el acondicionamiento físico, el desarrollo de habilidades y el juego. Por lo que la implantación de programas de formación orientados a estos objetivos puede ser beneficiosos a la hora de implementar estrategias que coadyuven a obtener una mayor salud de los niños y niñas mexicanos.

PALABRAS CLAVE: Programa de formación, México, Educación Primaria, Salud.

\section{Active physical education classes through sofit evaluation}

ABSTRACT: The purpose of this study was to implement a training program for 34 students of the Physical Activity and Sports Degree of the Sports School of the Autonomous University of Baja California (Mexico), in order to improve the activity time and intensity of the EF classes taught by these students. For the suitability of the training program, the variables intensity, index of moderate to vigorous physical activity and context were evaluated using the System for Observing Fitness Instruction Time (SOFIT; McKenzie, Sallis, y Nader, 1991). Following the training program, results, after giving $34 \mathrm{PE}$ sessions to school-aged children, were found to be above $50 \%$ moderate to vigorous intensity. Also, time was found to increase in tasks related to physical conditioning, skill development, and play. Therefore, the implementation of training programs aimed at these objectives can be beneficial when implementing strategies that contribute to obtaining better health for Mexican children.

KEY WORDS: Training program, Mexico, Primary Education, Health. 
Este artículo está publicado como capítulo en el libro: Hall, J. A., Ochoa, P. Y., y SáenzLópez, P. (2018). Intensidad, salud, motivación y adherencia en Educación Física. Huelva: Servicio de publicaciones de la Universidad de Huelva.

\section{INTRODUCCIÓN}

En la actualidad el sedentarismo es considerado como factor de riesgo independiente para padecer problemas de salud principalmente metabólicos y cardiovasculares (Pearce, Basterfield, Mann, Parkinson, y Adamson, 2012). Para prevenir y minimizar estos problemas se han hecho recomendaciones a nivel mundial para incrementar los niveles de actividad física, sin embargo, no parce ser suficiente (Katzmarzyk et al., 2008).

Investigaciones realizadas en México refieren que por cada hora de práctica de actividad física moderada a vigorosa los niños y adolescentes presentan un $10 \%$ menos de riesgo a padecer obesidad (Hernández et al., 1999), a su vez que el promedio de tiempo frente a pantallas de TV de los niños y adolescentes mexicanos es de 2.4 horas al día (Hernandez et al., 2000), atendiendo a que cada hora frente a estas se incrementa un $12 \%$ la prevalencia de obesidad (Hernández et al., 1999). De acuerdo con la última Encuesta Nacional de Salud y Nutrición (Morales-Ruan, Hernández-Prado, GomezAcosta, Shamah-Levy, y Cuevas-Nasu, 2009) el 40\% de los adolescentes mexicanos son sedentarios. Esta encuesta reporta que los niños de edad escolar (5 a 11 años), que la obesidad aumentó de $5.3 \%$ a $9.4 \%$ en niños y de $5.9 \%$ a $8.7 \%$ en niñas, del año 1999 al 2006. Asimismo, si se combina la presencia de sobrepeso y obesidad se aumentó de $18.6 \%$ a 26.3\% (Bonvecchio et al., 2009). De forma general, la magnitud de este problema no radica solo en su alta prevalencia sino con el riesgo a padecer enfermedades metabólicas y cardiovasculares (Ebbeling, Pawlak, y Ludwig, 2002).

Por su amplia cobertura, la educación básica es recomendada mundialmente para la promoción de actitudes positivas para desarrollar en los niños hábitos saludables de alimentación y actividad física como base de un estilo de vida saludable (Sallis y Glanz, 2009). En escuelas primarias mexicanas, se han aplicado programas de intervención que han sido efectivos al utilizar estrategias para prevenir factores de riesgo cardiovascular mediante componentes de hábitos saludables y actividad físicas (ColinRamirez et al., 2010; Perichart-Perera et al., 2008). Estrategias similares han dado resultados positivos disminuyendo factores de riesgo incrementando el gasto energético y disminuyendo el consumo calórico (Sallis y Glanz 2009).

De los componentes del gasto energético, la actividad física es el único que puede ser modificado de manera voluntaria (Honas et al., 2008). En concreto, en el ámbito escolar, las clases de educación física (EF) son el espacio ideal para fomentar la práctica de actividad física es el espacio ideal para su fomento (Story, Nanney, y Schwartz, 2009). De acuerdo con los estándares establecidos internacionalmente por la United States' National Association for Sport and Physical Education (NASPE, 2010) las clases de EF deben ser diseñadas para que el estudiante participe en actividades que, por lo menos, tengan una intensidad moderada a vigorosa por encima del $50 \%$ del tiempo de la clase, es decir, que estén activos con un gasto energético similar a caminar o correr (Banville, 2006). Asimismo, se han de establecer acciones pedagógicas que adhieran al estudiante en actividades divertidas, placenteras que induzcan la práctica de actividad física extraescolar (Erwin y Castelli, 2008). En México se han realizado investigaciones en escuelas Primarias, públicas y privadas, midiendo el impacto físico de las clases de EF utilizando como instrumento el System for Observing Fitness Instruction Time (SOFIT) de McKenzie, Sallis y Nader (1991). Estos datos se encuentran 
por debajo de los estándares internacionales recomendados largos (Pérez Bonilla, 2009; Jennings-Aburto et al., 2009).

De acuerdo a la secretaría de Educación Pública de México las clases de EF tiene una duración de 50 minutos por sesión. Los estudios de Pérez Bonilla (2009) y de Jennings-Aburto (2009) indicaron que el tiempo real de las clases de EF mostró una media de entre 37.3 y 39.8 minutos, respectivamente. Asimismo, los resultados de estas investigaciones mostraron que las clases de EF presentaron niveles de actividad física moderada y vigorosa entre un $29.2 \%$ (Jennings-Aburto et al., 2009) y un 38.2\% (Pérez Bonilla, 2009) del tiempo de práctica. Otros resultados indicaron que existía una gran cantidad de tiempo en que el alumnado permaneció parado, mientras se organizaban la clase, largas filas y tiempos de transición entre las actividades excesivos (Pérez Bonilla, 2009, Jennings-Aburto et al., 2009). Con respecto a este aspecto, Pérez Bonilla (2009) concluyen que estas bajas cotas de actividad, dentro de las clases, son el resultado a la cantidad de tiempo empleado en administrar y organizar las actividades relacionadas con: la capacitación de grupos, cambio de grupos o cambio de actividades dentro de la clase, no maximizando el tiempo en actividades en movimiento (Pérez Bonilla, 2009). Por otra parte, Jennings-Aburto et al (2009) encontraron que el alumnado durante los recreos practicaba actividad física a una intensidad con niveles de actividad física moderada y vigorosa de un $40 \%$.

En relación a los resultados encontrados en la literatura, el objetivo del presente estudio fue evaluar la intensidad, contexto, gasto calórico de la clase de EF antes y después de un programa de capacitación orientado a impartir clases de EF activas a estudiantes de la Licenciatura en Actividad Física y Deporte de la Escuela de Deportes de la Universidad Autónoma de Baja California (México). Para ello, en el presente estudio, se identifica como variable independiente la aplicación de un programa de capacitación orientado al aprendizaje para impartir clases de EF activas. Los cambios suscitados sobre las variables dependientes: intensidad, índice de actividad física moderada a vigorosa y contexto evaluada mediante el sistema, intensidad de la actividad física.

Proponiendo como hipótesis que, tras el término del programa de capacitación, se encontraran valores más altos de intensidad, índice de actividad física moderada a vigorosa y gasto calórico de los estudiantes de la licenciatura en actividad física y deporte participantes en las clases de EF posterior a la capacitación y a su vez se verá reflejado al en una segunda etapa impartir una clase de EF.

\section{MÉTODO}

\subsection{Muestra y selección de participantes}

La presente investigación fue llevada a cabo entre enero y mayo de 2012. El diseño del estudio fue cuasi-experimental longitudinal, relacionando la causa efecto al manipular la variable independiente y medir los efectos sobre las variables dependientes (Thomas, Nelson, Silverman, y Silverman, 2001).

La muestra estuvo compuesta por 34 alumnos de la Licenciatura en Actividad Física y Deporte de la Escuela de Deportes de la Universidad Autónoma de Baja California (México), que cursaban la asignatura de Evaluación de la Educación Física. Dichos participantes firmaron la carta de consentimiento atendiendo a los requerimientos de los principios éticos de investigación en seres humanos de la declaración de Helsinki (Puri, Suresh, Gogtay, y Thatte, 2009). A su vez el proyecto fue 
propuesto y aprobado por el comité de ética del programa de investigación y divulgación de la Escuela de Deportes de la Universidad Autónoma de Baja California (Protocolo n. 002/2012).

\subsection{Procedimiento}

El diseño metodológico consistió en cuatro fases. 1) La primera fase de la investigación fue establecer un diagnóstico inicial como punto de partida para evaluar las variables de: intensidad, índice de actividad física moderada vigorosa, contexto, frecuencia cardiaca y gasto calórico. Esta etapa, sin capacitación, fue de cuatro sesiones de EF, siendo ellos los propios alumnos. 2) La segunda fase consistió en una capacitación con contenidos sistema SOFIT, orientado a aplicar clases de EF con índices de actividad física de moderada a vigorosa. 3) La tercera fase fue solicitar nuevamente a los alumnos impartir 4 sesiones de EF, realizando la evaluación final de las variables de: intensidad, índice de actividad física moderada vigorosa, contexto, frecuencia cardiaca y gasto calórico. 4) La cuarta fase consistió en que cada uno de los estudiantes impartiera una clase EF a un grupo de 42 niños de edad escolar y evaluar las variables de intensidad, índice de actividad física moderada vigorosa y contexto. Para esta cuarta fase fue necesario solicitar la autorización de los directivos de la Casa Hogar Para Varones, AC (CAHOVA) de Mexicali Baja California (México), explicando por escrito el propósito de la investigación y el impacto, evaluando en total 34 sesiones de EF.

Los instrumentos empleados para la medición de las variables dependientes fueron:

System for Observing fitness and Instruction Time (McKenzie, Sallis, y Nader, 1991) sistema para observar el tiempo de instrucción de actividad física por sus siglas en ingles. Este permite evaluar las clases de EF eligiendo al azar a 4 estudiantes de cada clase para observarlos en secuencia rotatoria de 12 intervalos durante 20 segundos cada uno, repitiéndose las observaciones durante toda la clase, siguiendo el audio de SOFIT (McKenzie et al., 1991) se utilizó un reproductor MP3 Samsung YPU6AB. Para determinar la intensidad de la clase de EF se usan códigos para clasificar los niveles de actividad, los cuales permitieron estimar la energía gastada asociada con la actividad física, este procedimiento se ha usado para evaluar el tipo de actividad física relacionada con el currículum de EF, clasificando los códigos en cinco: 1) acostado, 2) sentado, 3) parado, 4) caminando, y 5) muy activo que corresponde a correr o a cuando el estudiante realiza más actividad física que la que corresponde al caminar ordinario. Suma porcentual de los códigos 4) caminando y 5) muy activo se obtiene un índice de actividad física de moderada a vigorosa (IAFMV). Estos códigos han sido calibrados monitoreando los latidos del corazón y el sistema ha sido validado usando acelerómetros Caltrac $\circledast$. A partir de la cuantificación de estos códigos se establece el índice de actividad física moderada a vigorosa se determinó sumando porcentualmente los códigos 4) caminando, y 5) muy activo del total del tiempo de la clase. Simultáneamente el sistema SOFIT evalúa el contexto de la clase el cual dirige el profesor de EF, en este apartado se identifican siete variables que se codifican de la siguiente manera: M) Contenido general, P) Conocimiento específico, K) Conocimiento general, F) Acondicionamiento físico, S) Desarrollo de habilidades, G) Juego, O) Otros.

El tiempo de la clase de EF se cronometro Casio HS3W estableciendo el inicio y fin de la clase de EF de acuerdo al horario establecido para la sesión de 50 minutos.

La intensidad de las clases de EF fue establecida por medio de la frecuencia cardiaca a través de pulsómetro Polar ${ }^{\circledR}$ FT7, determinando el promedio cada 5 minutos durante la clase y final durante la sesión de 50 minutos, determinando bajo el mismo 
instrumento y el gasto calórico promedio del total de la clase de los 4 estudiantes seleccionados a al azar que representaron la evaluación de los 12 intervalos durante 20 segundos en secuencia rotatoria, establecidos por SOFIT.

\subsection{Programa de capacitación}

A los 34 estudiantes participantes se les aplicó un programa de capacitación de 40 horas con contenidos de aprendizaje del sistema SOFIT, orientado a aplicar a las clases de EF. Los contenidos desarrollados versaron sobre los siguientes ejes temáticos 1) sedentarismo infantil. ¿cómo coadyuvar en la solución desde el ámbito escolar? 2) antecedentes de evaluación mediante el sistema SOFIT en escuelas primarias mexicanas 3) análisis evaluación de las clases impartidas en la primera fase de la investigación. Para estos contenidos previamente se grabaron las 4 sesiones de EF impartidas en la primera fase de la investigación, utilizando una cámara de video SONY handycam modelo DCR-SX40, reproduciendo las grabaciones y haciendo la retroalimentación especificando sobre el índice de actividad física moderada a vigorosa. El programa de capacitación se llevó a cabo durante dos meses con 3 sesiones por semana con un volumen semanales de 5 horas (dos días de dos horas de duración y un día de una hora).

\subsection{Análisis}

Para comprobar la hipótesis se propuso como análisis estadístico el cálculo de los porcentajes de cambio $(\Delta \%)$ para cada variable dependiente ([(Mediapost Mediapre) / Mediapre] x 100) (Vincent, 1999). También se utilizó estadística descriptiva para evaluar las variables del sistema SOFIT, en cuando los alumnos impartan la clase de EF en el ámbito escolar. Los resultados estadísticos se procesaron en forma de figuras y tablas en el software Microsoft Office Excel (ver. 2010).

\section{RESULTADOS}

En la Tabla 1, se encuentra el promedio de minutos utilizados en cuatro clases de EF impartidas por estudiantes de licenciatura en actividad física y deporte, antes y después de manipular la variable independiente respectivamente, utilizando como instrumento de evaluación: el sistema SOFIT.

Tabla 1. Duración en minutos de la intensidad y contexto utilizados en la importación de las clases de educación física Pre-capacitación y Post-capacitación.

\begin{tabular}{ccc}
\hline \multirow{2}{*}{ Variables } & \multicolumn{2}{c}{ Duración en minutos de la evaluación SOFIT } \\
\cline { 2 - 3 } & Pre-capacitación & Post-capacitación \\
\hline 1) Acostado (min) & 0 & 1.8 \\
2) Sentado (min) & 2.1 & 2.1 \\
3) Parado (min) & 27.6 & 15.1 \\
4) Caminando (min) & 12.5 & 18.5 \\
\hline
\end{tabular}




\begin{tabular}{lcc}
\hline 5) Muy activo (min) & 8.1 & 12.5 \\
M) Contenido general (min) & 19.1 & 13 \\
P) Conocimiento específico (min) & 0 & 1.1 \\
K) Conocimiento general (min) & 0 & 1.3 \\
F) Acondicionamiento físico (min) & 5.9 & 11.8 \\
S) Desarrollo de habilidades (min) & 5.2 & 10.1 \\
G) Juego (min) & 17.7 & 10.3 \\
O) Otros (min) & 2.1 & 2.4 \\
\hline
\end{tabular}

En la Figura 1 se muestra la variable independiente respectivamente antes y después de la aplicación del programa de capacitación las a cuatro clases de EF impartidas por estudiantes.

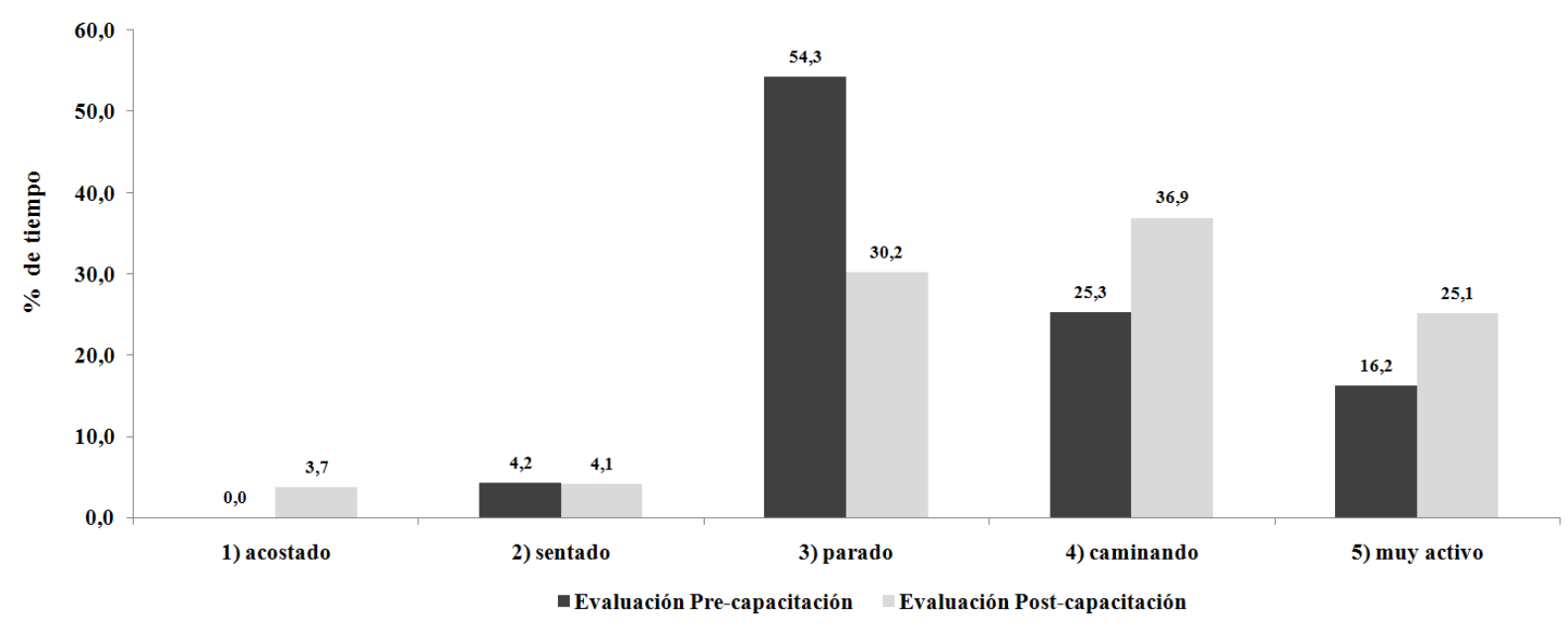

Figura 1. Distribución porcentual de la intensidad de actividad física de la clase de educación física impartidas por los alumnos de licenciatura en actividad física y deporte evaluada Precapacitación y Post-capacitación.

En la Figura 2 se muestra que el índice de actividad física de moderada a vigorosa (IAFMV) fue mayor tras la aplicación del programa de capacitación. 


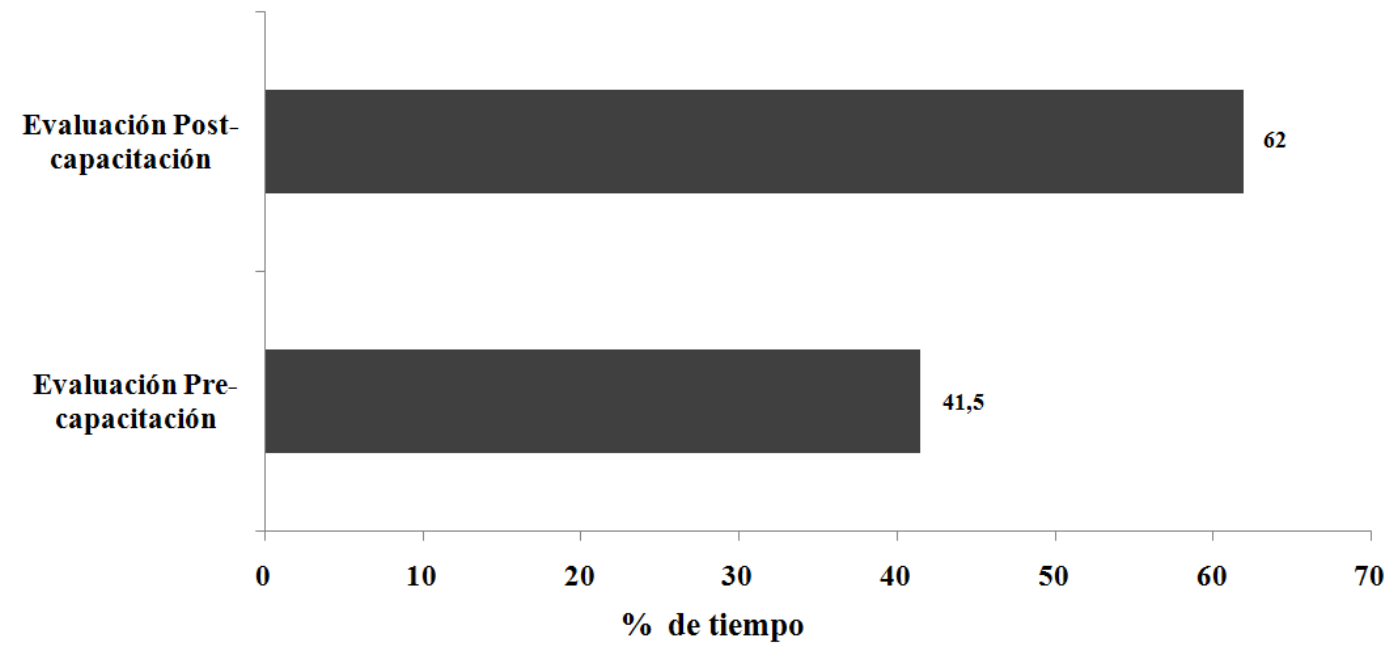

Figura 2. Distribución porcentual del índice de actividad física moderada a vigorosa IAFMV de las clases de educación física impartidas por los alumnos de licenciatura en actividad física y deporte evaluada Pre-capacitación y Post-capacitación.

Las sesiones de EF correspondientes a cuatro clases de EF impartidas por estudiantes de licenciatura en actividad física y deporte, antes y después manipular la variable independiente respectivamente, induciendo un mayor uso de actividades relacionadas con el acondicionamiento físico y el desarrollo de habilidades (ver Figura 3).



Figura 3. Distribución porcentual de la intensidad del contexto de las clases de educación física impartidas por los alumnos de licenciatura en actividad física y deporte evaluada Pre-

capacitación y Post-capacitación

En la siguiente figura (Figura 4), se muestra la intensidad de la frecuencia cardiaca. Esta muestra ser superior durante todas las mediciones tomadas, excepto el último tramo (45 a 60 minutos) tras la aplicación del programa de capacitación. 


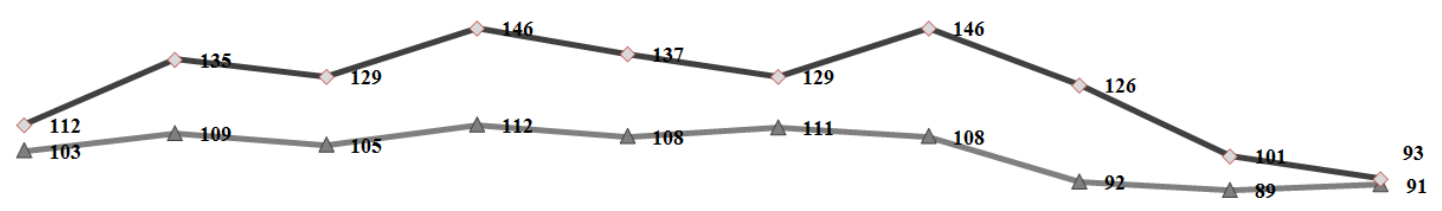

5 10 15 20 25 30 35 40 45

Figura 4. Frecuencia cardiaca promedio medida en intervalos de 5 minutos de las clases de educación física impartidas por los alumnos de licenciatura en actividad física y deporte evaluada Pre-capacitación y Post-capacitación.

En cuanto los resultados obtenidos tras la capacitación, se impartieron 34 sesiones de EF en el ámbito escolar a niños de edad escolar cuantificando el IAFMV. Este índice mostró que el $57 \%$ del tiempo de clase, el alumnado se encontraba en movimiento. Más concretamente, se muestra en la Figura 5 que el $21 \%$ del tiempo el alumnado se encontraba muy activo.

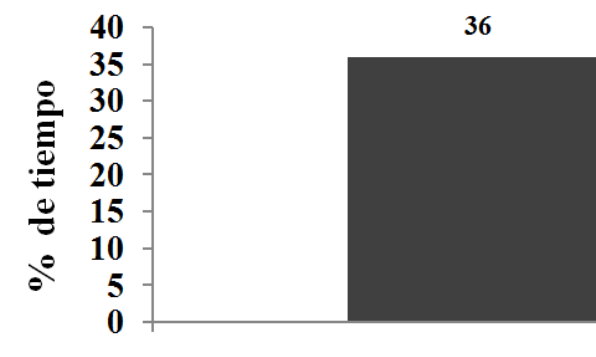

4) caminando
21

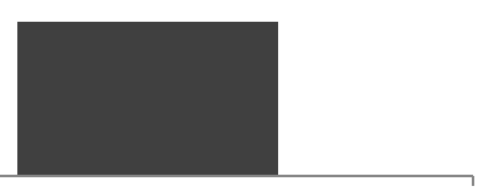

5) muy activo

Figura 5. Distribución porcentual de los indicadores para el índice de actividad física moderada a vigorosa IAFMV de las clases de educación física impartidas por los alumnos de licenciatura en actividad física y deporte en el ámbito escolar Post-capacitación.

Por último, los contenidos mayormente usados para las clases de EF fueron los encaminados a la transmisión de contenidos generales, acondicionamiento físico y el juego, principalmente (Figura 6). 


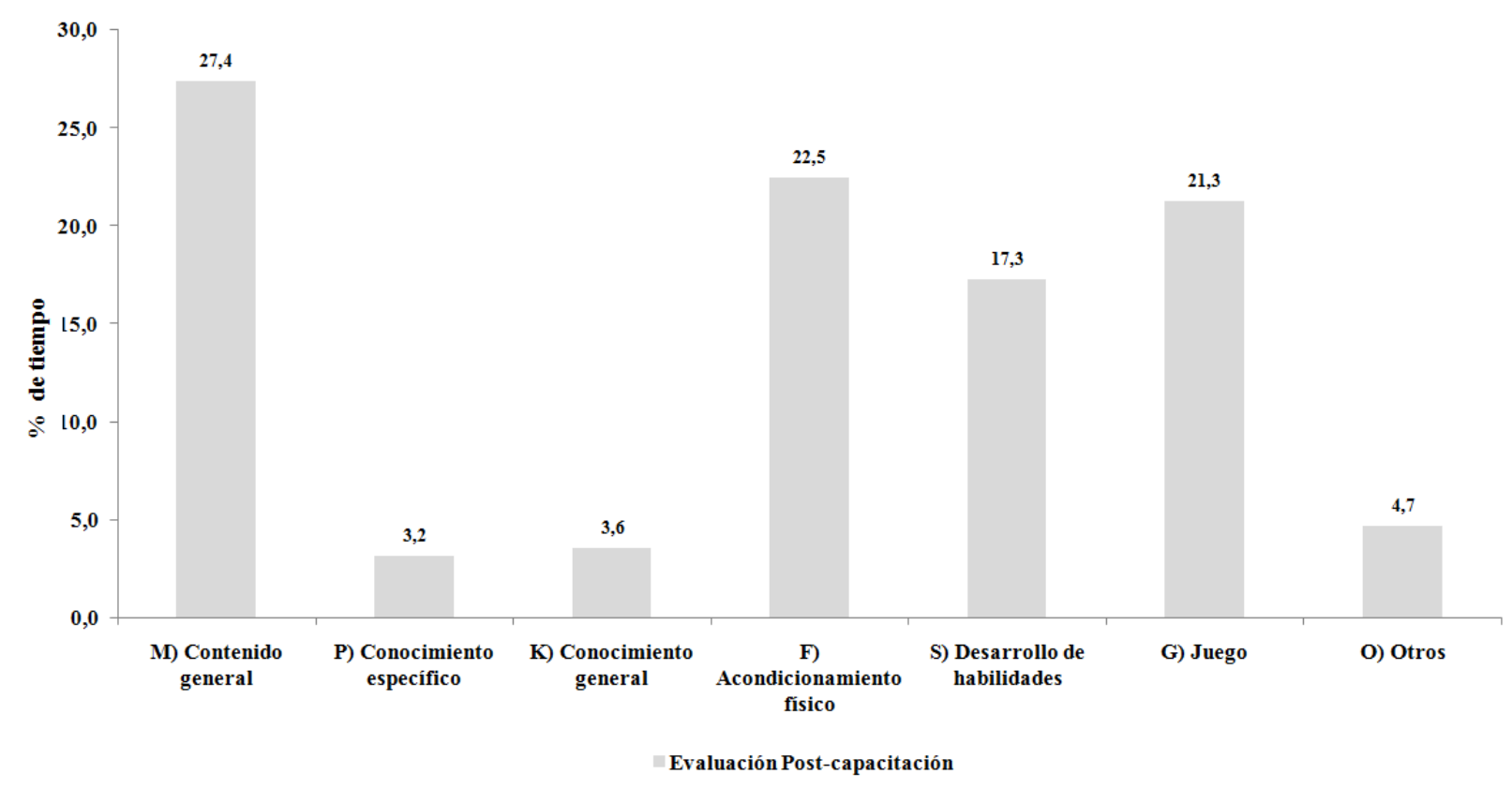

Figura 6. Distribución porcentual de la intensidad del contexto de las clases de educación física impartidas a niños de dad escolar por los alumnos de licenciatura en actividad física y deporte Post-capacitación.

\section{DISCUSIÓN Y CONCLUSIONES}

El presente estudio tuvo como finalidad instruir a futuros docentes los cuales en corto plazo estarán en servicio, y conocer los resultados de aplicabilidad para impartir clases de EF activa, tomando en cuenta el sedentarismo infantil y teniendo como oportunidad el ámbito escolar para su solución. Tras analizar las investigaciones que refieren el trabajo actual del profesorado de EF, en cuanto a la impartición de clases activas así como análisis evaluación de las clases impartidas pre-capacitación, donde pudimos reflejar los resultados en las 34 sesiones de EF impartidas por estudiantes de licenciatura en actividad física y deporte, a niños de edad escolar, y post-capacitación, encontrando un intensidad moderada a vigorosa por arriba del $50 \%$, cubriendo los indicadores de intensidad 4) caminando 5) muy activo con un promedio porcentual de $36 \%$ y $21 \%$ respectivamente, incorporando estrategias didácticas en las sesiones donde se distribuyó el contexto disminuyendo así el tiempo empleado para M) Contenido general, al administrar eficientemente el tiempo y organizar las actividades relacionadas con instrucciones tales como la capacitación de equipos, cambio de equipos o cambio de actividades dentro de la clase y aprovecharlas para F) Acondicionamiento físico, S) Desarrollo de habilidades y G) Juego.

De acuerdo con los estándares establecidos internacionalmente por el United States' National Association for Sport and Physical Education (NASPE), uno de los parámetros importantes para impartir clases de EF activas es que los profesores deben diseñar sesiones en los que el estudiante participe en actividades que tengan una intensidad moderada a vigorosa por encima del $50 \%$ del tiempo real de clase, es decir que estén activos con un gasto energético similar al de caminar o correr (Banville, 2006). Los resultados del presente estudio muestran indicadores de intensidad, evaluado por el instrumento SOFIT, en los cuales los alumnos de Licenciatura en Actividad Física y Deporte incrementaron el índice de actividad física moderada a vigorosa del $41.5 \%$ precapacitación al $62 \%$ post-capacitación, con un porcentaje de cambio $(\Delta \%)$ de $48.8 \%$. 
En México ya existen trabajos donde se ha usado el sistema SOFIT en clases de EF, obteniendo índices de actividad física moderada y vigorosa de $29.2 \%$ (JenningsAburto et al., 2009) y 38.2\% (Pérez Bonilla, 2009) los cuales fueron porcentajes similares a los en encontrados en las clases de EF impartidas pre-capacitación. Asimismo, los resultados de intensidad se evaluaron bajo otro instrumento como fue la telemetría mediante pulsómetro. Estos determinaron que el promedio de frecuencia cardiaca y gasto calórico los cuales fueron mayores tras la capacitación incrementando de 103 latidos por minuto a 125 latidos por minuto en cuanto a la frecuencia cardiaca. El gasto calórico se incrementó de 245 kilocalorías a 342 kilocalorías promedio de las clases de EF post-capacitación, ambas variables representaron un porcentaje de cambio $(\Delta \%)$ de $21.4 \%$ y $39.6 \%$ respectivamente.

Al analizar los resultados de las dos investigaciones referentes en México (Jennings-Aburto et al 2009; Pérez Bonilla, 2009), donde se utilizó en el diseño metodológico la evaluación SOFIT, se encuentran resultados en los cuales el profesorado de EF no utilizó la totalidad del tiempo para administrar la clase de EF, impartiendo en promedio 37.3 minutos (Pérez Bonilla, 2009) y 39.8 minutos (JenningsAburto et al 2009) del total de los 50 minutos. Además, se establece el tiempo al que se dedica el tiempo de clase como es el contexto de M) Contenido general, el cual incluye actividades como la transición, administración y descanso de la clase, en donde los niños permanecían la mayor parte del tiempo en intensidades como: 1) acostado, 2) sentado, 3) parado, lo cual no provoca un gasto energético relevante. En ese sentido la formación del profesorado tiene una influencia que puede aportar a la salud física del alumno durante las clases de EF (Story et al., 2009).

Con los resultados obtenidos puede afirmarse que se logró incrementar la intensidad modificando el contexto Este programa implementado ayudó a maximizar el uso del tiempo de la clase de EF e implementar estrategias que permitan mantener a los estudiantes en intensidades moderadas a vigorosas por lo menos el $50 \%$ de la clase. Por ello consideramos que programas formativos como este pueden implementarse en los programas de licenciatura de México. Sin embargo, es necesario poder aplicar futuras investigaciones con un control mayor de la variable independiente. Asimismo, se plantea la necesidad de investigaciones futuras donde se incorporen programas de capacitación en docentes en servicio, estratificar las evaluaciones por sexo, ampliar la cantidad de tiempo en la evaluación, utilizar estadística inferencial y ampliar la muestra. De forma general, se plantea la necesidad de proseguir en esta línea de investigación para poder crear recomendaciones que puedan servir de referencia al profesional de la EF con el objeto de tener mayor número de herramientas que permitan mejorar la actuación docente e implementar estrategias que coadyuven desde el ámbito escolar el obtener una mayor salud de los niños a través del movimiento.

\section{REFERENCIAS}

Banville, D. (2006). Analysis of exchanges between novice and cooperating teachers during internships using the NCATE/NASPE Standards for Teacher Preparation in Physical Education as guidelines. Research Quarterly for Exercise and Sport, 77(2), 208-221. doi: 10.1080/02701367.2006.10599355

Bonvecchio, A., Safdie, M., Monterrubio, E. A., Gust, T., Villalpando, S., y Rivera, J. A. (2009). Overweight and obesity trends in Mexican children 2 to 18 years of age from 1988 to 2006. Salud Pública de México, 51 Suppl 4, S586-594. doi: $10.1590 / \mathrm{s} 0036-36342009001000013$ 
Colin-Ramirez, E., Castillo-Martinez, L., Orea-Tejeda, A., Vergara-Castaneda, A., Keirns-Davis, C., y Villa-Romero, A. (2010). Outcomes of a school-based intervention (RESCATE) to improve physical activity patterns in Mexican children aged 8-10 years. Health Education Research, 25(6), 1042-1049. doi: 10.1093/her/cyq056

Ebbeling, C. B., Pawlak, D. B., y Ludwig, D. S. (2002). Childhood obesity: public-health crisis, common sense cure. Lancet, 360(9331), 473-482. doi: 10.1016/S01406736(02)09678-2

Erwin, H. E., y Castelli, D. M. (2008). National physical education standards: a summary of student performance and its correlates. Research Quarterly for Exercise and Sport, 79(4), 495-505. doi: 10.1080/02701367.2008.10599516

Hernández, B., Gortmaker, S.L., Colditz, G.A., Peterson, K.E., Laird, N.M, y ParraCabrera, S. (1999) Association of obesity with physical activity, television programs and other forms of video viewing among children in Mexico City. International Journal of Obesity and Related Metabolic Disorders, 23(8):845-54. Doi: $10.1038 /$ sj.ijo.0800962

Hernández, B., Gortmaker, S. L., Laird, N. M., Colditz, G. A., Parra-Cabrera, S., y Peterson, K. E. (2000). Validity and reproducibility of a questionnaire on physical activity and non-activity for school children in Mexico City. Salud Pública de México, 42(4), 315-323.

Honas, J. J., Washburn, R.A, Smith, B.K., Greene, J.L., Cook-Wiens, G., y Donnelly. J. E. (2008). The System for Observing Fitness Instruction Time (SOFIT) as a measure of energy expenditure during classroom-based physical activity. Pediatric Exercise Science, 20(4), 439-445. doi: 10.1123/pes.20.4.439

Jennings-Aburto, N., Nava, F., Bonvecchio, A., Safdie, M., Gonzalez-Casanova, I., Gust, T., y Rivera, J. (2009). Physical activity during the school day in public primary schools in Mexico City. Salud Pública de México, 51(2), 141-147. doi: 10.1590/s0036-36342009000200010

Katzmarzyk, P. T., Baur, L. A., Blair, S. N., Lambert, E. V., Oppert, J. M., Riddoch, C. The Conference Speaker, P. (2008). International conference on physical activity and obesity in children: summary statement and recommendations. International Journal of Pediatric Obesity, 3(1), 3-21. doi: 10.1080/17477160701789679

McKenzie, T., Sallis, J., y Nader, P. (1991). SOFIT. System for Observing Fitness Instruction Time. Journal of Teaching in Physical Education,11(2), 195-205.

Morales-Ruan M. C., Hernandez-Prado, B., Gomez-Acosta, L. M., Shamah-Levy, T., y Cuevas-Nasu, L. (2009). Obesity, overweight, screen time and physical activity in Mexican adolescents. Salud Pública de México, 51 Suppl 4, S613-620. doi: 10.1590/s0036-36342009001000016

Pearce, M. S., Basterfield, L., Mann, K. D., Parkinson, K. N., y Adamson, A. J. (2012). Early predictors of objectively measured physical activity and sedentary behaviour in 8-10 year old children: the Gateshead Millennium Study. PLoS One, 7(6), e37975. doi: 10.1371/journal.pone.0037975. 
Pérez Bonilla, A.M. (2009) Impacto de la clase de educación física sobre la actividad moderada y vigorosa en niños de primaria. Revista Mexicana de Investigación en Cultura Física y Deporte, 1(1),150-172,

Perichart-Perera, O., Balas-Nakash, M., Ortiz-Rodríguez, V., Morán-Zenteno, J.A., Guerrero-Ortiz, J.L., y Vadillo-Ortega, F. (2008) A program to improve some cardiovascular risk factors in Mexican school age children. Salud Pública de México, 50(3):218-226. doi: 10.1590/s0036-36342008000300005

Puri, K. S., Suresh, K. R., Gogtay, N. J., y Thatte, U. M. (2009). Declaration of Helsinki, 2008: implications for stakeholders in research. Journal of Postgraduate Medicine, 55(2), 131-134. doi: 10.4103/0022-3859.52846

Sallis, J. F., y Glanz, K. (2009). Physical activity and food environments: solutions to the obesity epidemic. Milbank Quarterly, 87(1), 123-154. doi: 10.1111/j.14680009.2009.00550.x

Secretaría de Educación Pública (n.d.). Retrieved may 16, 2010, from the Plan de Estudios 2009 para Educación Básica. Primarias. Etapa de Prueba, México; 2009. Population website, http://efmexico.wordpress.com/apoyos-didacticospara-la-clase-de-ef/programas-para-educacion-basica/

Subsecretaría de Educación Básica. Dirección General de Desarrollo Curricular. Subdirección de Educación Física (n.d.). Retrieved may 12, 2010, from the Guía de Educación Física para la Educación Primaria, México; 2008. Population website, http://efmexico.files.wordpress.com/2008/08/guia_primarias_piloto.pdf

Story, M., Nanney, M. S., y Schwartz, M. B. (2009). Schools and obesity prevention: creating school environments and policies to promote healthy eating and physical activity. Milbank Quarterly, 87(1), 71-100. doi: 10.1111/j.14680009.2009.00548.x

Thomas, J.R., Nelson, J.K., Silverman, S., y Silverman, S.J. (2001). Research Methods in Physical Activity (6th. Ed.). Champaign, Ilinois: Human Kinetics.

United States' National Association for Sport and Physical Education NASPE (2009). data revisited. (n.d.). Retrieved may 9, 2009, from the national standards guidelines and position statements, E.U.A.. Population website,http://www.aahperd.org/naspe/

Vincent, W. J. (1999) Statistics in kinesiology (2nd Ed.). Champaign, Ilinois: Human Kinetics. 